\title{
Physiological and psychological effects of forest healing focused on plant fragrance therapy for maladjusted soldiers
}

\author{
Jihye Kim ${ }^{1}$, Changseob Sin ${ }^{2}$, Jong-ok Park ${ }^{3}$, Hyunchae Lee ${ }^{4}$, Jihye Kim ${ }^{4}$, Dohyeong Kim ${ }^{5}$, and Sanghee Kim 6 \\ ${ }^{1}$ Doctoral student, Graduate Department of Forest Therapy, Chungbuk National University, Cheongju 28864, Korea \\ ${ }^{2}$ Professor, Department of Forest Science, Chungbuk National University, Cheongju 28864, Korea \\ ${ }^{3}$ Colonel, Republic of Korea Army, Korea \\ ${ }^{4}$ Researcher, leumforest Inc., Seoul 05315, Korea \\ ${ }^{5}$ Major, Republic of Korea Army, Korea \\ ${ }^{6}$ Ph.D., Integrated medicine Lab., Sunmoon University, Asan 31460
}

\section{ABSTRACT}

Background and objective: This study verified the physiological and psychological effects of plant fragrance therapy to analyze whether the therapy has a forest healing effect on maladjusted soldiers.

Methods: This study was conducted from March 14 to May 31, 2020 according to the Green Camp curriculum for 2 weeks per recruit class. Thus, plant fragrance therapy was implemented twice each for three recruit classes. Each session of the program was carried out for 4 hours from 1 p.m. to 5 p.m. The venue was the garden within the camp and the forest nearby. Forest healing activities using plant fragrances were led by 1 forest education specialist and 1 assistant. The effects of plant fragrance therapy for maladjusted soldiers were analzed analyzed by quantifying physiological and psychological changes through measuring brain waves and stress levels before and after the therapy.

Results: As a result of analyzing BRQ and SQ to determine the physiological and psychological healing effect of plant fragrance therapy, both the left and right brain showed higher BRQ and SQ after the therapy with statistical significance. This indicates that plant fragrance therapy on maladjusted soldiers helped positively stabilize their negative psychological and behavioral dispositions, and stabilization of brain waves lowered physical and mental stress and improved self-regulation and immunity. On the other hand, there was no significant difference in ATO. This is the result of analyzing the stress-coping mechanism. Troubleshooting $(\mathrm{t}=-2.702, \mathrm{df}=61, p=.009)$, emotional remission $(\mathrm{t}=-2.173, \mathrm{df}=61$, $p=.034)$, pursuit of help $(t=-3.286), d f=61, p=.002)$, and wishful thinking $(t=-3.714, d f=61, p<.001)$ showed statistically significant results.

Conclusion: Applying plant fragrance therapy to maladjusted soldiers positively stabilized their negative psychological and behavioral dispositions and helped their self-regulation. Thus, plant fragrance therapy has psychological and physical forest healing effect on maladjusted soldiers.

Keywords: fragrance, plant, therapy, mental health, brain wave

\section{Introduction}

Plants that form the forest contain many bioactive compounds that are beneficial for health (Kris-Etherton PM et al., 2002). Over 50,000 plant species are used for medicinal purposes around the world (Schippmann et al., 2006), and about $17 \%$ of vascular plants worldwide belong to plant species used by humans (Hamilton et al., 2006). The WHO listed 21,000 species of plants used for medical purposes (Groombride, 1992). 122 compounds can be obtained from 94 species of plants that are widely used as medicine. The active elements in the compounds of these plants are at

This research program was supported by the Korea Forest Service's green funding project, the Social Value Promotion Project.

Received: April 7, 2021, Revised: April 30, 2021, Accepted: July 13, 2021

First author: Jihye Kim, ieumforest@gmail.com, (10) https://orcid.org/0000-0002-2004-1665

*Corresponding author: Jong-ok Park, ieumforest@ieumforest.or.kr, (1D) https://orcid.org/0000-0001-9533-9055 
least $80 \%$ identical to the information used in human drugs and thus are used for medical purposes (Fabricant and Farnsworth, 2001).

Forests have phytoncide, a commonly known biologically active substance, that has positive physical and psychological effects for people to cope effectively with stress (Mass et al., 2009).

Phytoncide refers to the fragrance of the forest and is a volatile chemical compound of a fragrance released by trees. Phytoncide is a Greek word combining 'phyton' meaning 'plant' and 'cide' meaning 'kill, sterilize'. This substance enters the body and eliminates germs, pests, and fungi, having a beneficial effect on the human body and making us feel comfortable. Phytoncide is a volatile substance created and released by trees, and the main component is an organic compound called terpene. Trees use solar energy with photosynthesis to generate carbohydrate from water and carbonic acid gas and release oxygen, secondarily creating phytoncide to protect themselves. Aroma oil from plants is inhaled through the nose and delivered to the olfactory nerves, after which it is directly transmitted to the brain through the limbic system of the cerebrum. Aromatherapy through the limbic system stimulates the olfactory nerves of the olfactory bulbs when the aroma is inhaled, and then delivered to each part of the brain. This exerts a therapeutic effect on the autonomic nervous system, endocrine regulation of the immune system, and physical diseases and symptoms (Kim, 2011). If we take a forest bath in the forest where terpene is volatilized, phytoncide enters our body, eliminates bad pathogens through the lymphatic system, and increases immunity to maintain psychological and physical health. This can help natural therapy using the unique volatile organic compounds of forest fragrances relieve stress and prevent diseases of people today as total care (Song, 2015). Since fragrances of the forest affects the cerebral cortex and hypothalamus and therefore have a therapeutic effect on the whole body, inhaling a pleasant fragrance in a stressed condition will affect the limbic system of the cerebrum that is close to the basic instincts such as anxiety, fear, and rage, thereby relieving stress and changing the person into a stable state so that they can cope positively with stress (Kim et al., 2020).

Previous studies on the effect of volatilized organic com- pounds like phytoncide on the human body proved that phytoncide physically and mentally helps humans. Similar immunoregulatory effects were found in several pimarane diterpenoids separated from the lumber and leaves of Tetraclinis (Barrero et al., 2003), and sandarac resins extracted from the Cupressus genus Tetraclinis and Callitris are used to treat hypertension, heart diseases, skin diseases, and neurological disorders such as depression (Ait Igri et al., 1990).

Phytoncide also lowers the levels of life stress and stress hormones in blood by inhaling it (Nam et al., 2008). The human body after a forest bath showed stable brain waves and blood pressure as well as relaxed pulsation, and the decreased stress resulted in physiological change and psychological stability (Lee, 2012).

Studies on plant-derived essential oils revealed that antibacterial activity and immunoregulation of terpene in volatile essential oils of plants are beneficial to the human body (Buhagiar et al., 2000). Essential oils also emotionally, mentally, and physically improved the mental state such as stress, depression, and anxiety, and physical treatment and inhaling of aroma relieved or prevented stress (Jun, 2007). Essential oils from fragrant plant bodies promote mental and physical health and are applied as complementary and alternative therapy to prevent and treat various diseases (Ha, 2006).

Forest healing refers to immune-strengthening and health-promoting activities, which utilize a variety of elements of the forest, including scents and scenic views (Article 2 (4) of the Forestry Culture and Recreation Act). Forests are comprised of various environmental stimulating factors such as fragrances, sounds of birds singing and water flowing, and various colors of nature, which make forest healing activities more effective in reducing physical and mental stress, while also physiologically lowering stress levels and improves stress resistance (Park et al., 2014). Moreover, stress reactions can be adjusted by changing the situations or negative emotions of individuals or managing physical responses (Kogan and Betrus, 1984), and forest experience serves as a positive factor in improving the mood and relieving the stress of protective-concerned soldiers and general soldiers (You and Shin, 2017).

Forest healing programs using plant fragrances are part 
of the five senses that are necessary for classification of forest healing factors (Korea Forest Service, 2011). Plant fragrances can provide a positive effect when maladjusted soldiers are facing stressful situations, and forest healing programs relieved psychological and physiological stress reactions, improved maladaptive behavior of soldiers caused by stress, and reduced cortisol levels (Kim et al., 2020). The positive change of stress relief also served as a direct factor of adjustment to military life (Joe, 2003), and thus forest healing activities using plant fragrances for maladjusted soldiers will help treat and prevent their stress (Kim et al., 2020).

Previous studies on maladjustment to military service implied that soldiers had maladjustment factors caused by stress, and their stress was related to brain functions (S. Larsen, 2012; J. Robbins, 2000). Moreover, due to the special environment of the military, private and private first-class soldiers are suffering greater stress from not being able to express their personal opinions or having freedom of action (Jung, 2007). This stress causes psychological maladjustment (Jung and Lee, 2013), which decreases coping skills (Lazarus and Folkman, 1984) and self-esteem (Lee, 2003). These results have limitations in that most studies used self-report tests in the psychological domain, while there were very few studies on the physiological domain.

To overcome the limitations of previous studies and analyze whether forest healing activities using plant fragrances can be an effective alternative therapy for maladjusted soldiers, this study applied these activities in two sessions, once a week, to Green Camp soldiers and examined various effects in the physiological and psychological aspects.

\section{Research Methods}

The goal of this study is to help Green Camp soldiers prevent and manage their stress and make plans for the future by improving self-awareness stress coping skills, positive emotional experience, and self-esteem considering their characteristics before and after plant fragrance therapy (or plant scent therapy/plant aromatherapy). Forest healing activities using plant fragrances were carried out with reference to previous studies that are related to stress relief and aromatherapy for the subjects. The activities were planned according to their goals based on accessibility to plant fragrances, seasonal elements of the forest, and composition of surrounding plants, designed to use plant fragrances by measuring individual brain waves (Fig. 1).

\section{Methods}

This study was conducted from March 14 to May 31, 2020 according to the Green Camp curriculum for 2 weeks per recruit class. Thus, plant fragrance therapy was implemented twice each for three recruit classes. Each session of the program was carried out for 4 hours from 1 p.m. to 5 p.m. The venue was the garden within the camp and the forest nearby. Forest healing activities using plant fragrances were led by 1 forest education specialist and 1 assistant. The soldiers were to have only limited access to cigarettes and caffeine while participating in the program. The main host of the program was a forest welfare specialist who received specialized training for aromatherapy and brain wave analysis. The program was carried out in the form of individual measurement of participants, counseling and application of plant fragrances, and prevention and management. Plant fragrance therapy was comprised of ac-

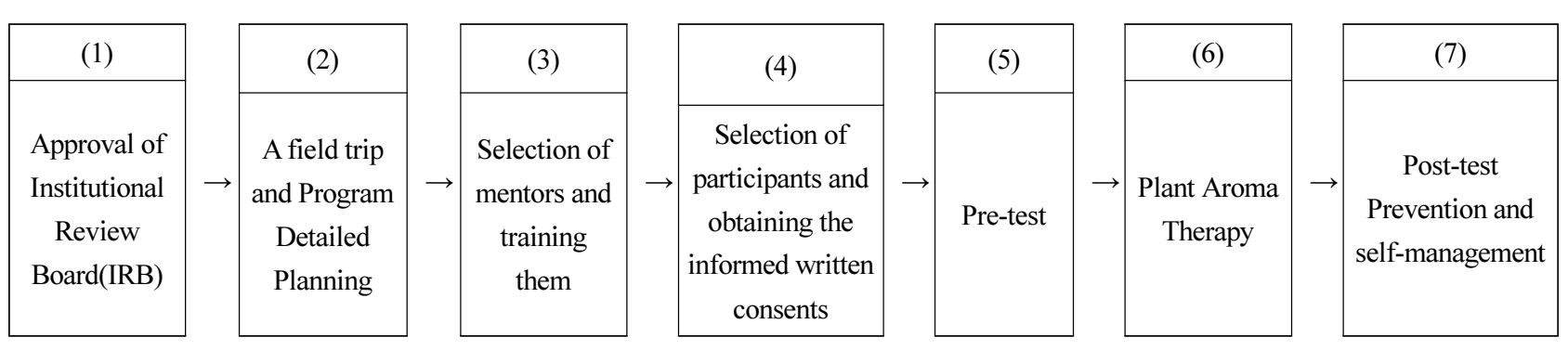

Fig. 1. Research design. 
tivities such as finding the right plant fragrances for each individual, experiencing plant fragrances, making their own perfume, forest trekking, making plant oil, and healing care, which were assisted by 4 squad leaders of Green Camp who received training in advance. The program was carried out from 1 p.m. to 5 p.m. to increase the therapeutic effect by making the best use of volatile fragrances of coniferous tree species at 15-23 degrees, the average temperature of March - May (Eddouks et al., 2002).

The venue for the program can be divided into the coniferous forest zone and deciduous forest zone. The former is a mixed forest dominated by coniferous trees showing 10 species such as artificially reproduced Pinus koraiensis, P. rigida, P. densiflora, Retinispora, and Cupressus. The latter shows 11 species including Castania crenata, Larix kaempferi, Quercus variabilis, P. jamasakura, artificially reproduced Rhododendron mucronulatum Turcz. var. mucronulat, and Hibiscus tiliaceus.

Plant fragrance therapy in this study is carried out by first measuring brain waves of individual participants to identify the stress levels and physiological and psychological changes. Then, the participants are to smell the fragrances of various plant-derived essential oils and fill out the test form. Among the essential oils authorized for aromatherapy, they choose the ones they prefer or are suitable for their physical and mental state, while also checking their current health. After they check their favorite fragrance type and plant that helps their mind and body, they learn the mechanism in which each plant fragrance response to the body. After that they go to the forest within the camp for trekking and forest bathing so that they can sufficiently absorb the plant fragrances using the main vegetations in the forest. To inhale the fragrances that are suitable for each individual soldier as identified in advance, they were to make and use perfume or massage oil they can actually use in everyday life. By learning how to easily use plant fragrances, they can improve their mental and physical health as well as immunity, reduce their stress, and take preventive measures. This program induces participants to create a synergy in mental and physical health by learning how to use plant fragrances and forest healing factors depending on their condition.

\section{Subjects}

In this study, maladjusted soldiers are soldiers who need treatment and care and are not appropriate for military service, referred to as 'protective-concerned soldiers' that are likely to cause accidents in the military camp and require isolation. They are soldiers classified by the standard for the military life support system (Article 232-2 Unit Management Directive, Ministry of National Defense Directive No. 2273). The army established Green Camp in July 2011 to provide care and treatment for maladjusted soldiers and suicidal soldiers so that their suicide can be prevented and they can be committed to combat mission. The camp is operated with a two-week program set up at the corps level, and follow-up management of soldiers is determined by the release deliberation procedures once a month. The soldiers participating in this study also participated in the two-session program according to the admission and release schedule of Green Camp at the T Corps in Gangwon-do. Soldiers with spastic neurological diseases, severe allergic reactions, immunologically serious illnesses, and limited activity due to trauma were excluded, leaving ultimately 62 soldiers participating in the program divided into three groups with one recruit class per month. They were soldiers recommended by their unit who voluntarily participated in the purpose of this study and explanation of the method. The average age of the participants was $22.84 \pm 2.0323$ years. The rank of each group was: 28 private first class soldiers $(45.2 \%), 20$ corporals $(32.3 \%)$, and 14 sergeants $(22.5 \%)$.

\section{Analytical tools}

\section{Brain wave measurement}

Since German medical scientist Hans Berger first measured brain waves of humans in 1924, it has become possible to treat mental problems of people today caused by stress, all kinds of addictive diseases, hormone and endocrine disorders, and immunologic dissonance. Brain waves are used in clinical trials or studies of mental and neurological disorders that can be observed continuously and noninvasively in the physiological and functional aspect on a real-time basis by brain activity of individuals. Accordingly, this study measured the brain waves that 
change on a real-time basis with individual brain activity when inhaling fragrances and identified the changes in brain waves before and after plant fragrance therapy. NeuroHarmony S portable 2-channel neurofeedback system measuring brain waves from prefrontal lobes by Panaxtos was used to compare the brain waves before and after the program, deriving the overall brain functions such as energy as well as brain psychology. This is a frequency spectrum analysis method to identify the level by correlation. Moreover, we comparatively measured brain waves with eyes open and eyes closed to determine the frequency and measured self-regulation of brains with NeuroHarmony S (Bak, 2007).

The reliability and validity of NeuroHarmony S portable 2-channel neurofeedback system is verified by previous studies with the correlation coefficient of $.916(p<.001)$ for $\alpha, \beta$, and $\theta$ waves on the left and right compared to the US Grass Neurodata Amplifier System (Kim, 2000). Brain waves refer to the total of consistent voltage that occurs after synapse of pyramidal cells in the cortex when information is exchanged among neurons, providing information about the electrical activity of the brain. For the frequency band, standard bands such as $\theta$ wave $(4-7 \mathrm{~Hz})$, $\alpha$ wave (8-12Hz), SMR (13-16Hz), $\beta$-low (13-20Hz), $\beta$ -hi (21-30Hz) were applied. SMR (Sensor-Motor Rhythm) refers to brain waves discovered by Dr. M. B. Sterman from UCLA (University of California, Los Angeles), indicating the rhythm discovered in the sensor cortex and motor cortex among the cerebral cortexes, representing the level of brain awakening. By printing these brain wave signals as digital signals, the simple noninvasive method is used. Different brain waves are correlated with the level of conscious awakening and experience, and brain waves strengthens the environment and protect the brain from hippocampal atrophy in the chronic brain damage stage (L.S. Miller et al., 2013). Moreover, people with ADD (attention deficit disorder) or ADHD (attention deficit hyperactivity disorder) can watch the changes in their brain waves on a screen and increase SMR (Sensor-Motor Rhythm) and low- $\beta$ to reduce brain waves related to impulsivity (S.Larsen, 2012). Brain waves also carefully refine sensory stimulations and enable people to regulate their senses on their own (S.Larsen, 2012; J.Robbins, 2000). Changes can be verified by measuring and analyzing brain waves of maladjusted soldiers before and after the program. We can also determine the psychological and physical stability and tension as well as self-regulation and stress with changes in alpha waves. This study showed the calculations of dispersity and ratio among frequency bands of brain waves in quotients and classified the frequency quotients of each brain function and presented them in pretest and posttest ratios. Here, the quotients are classified into three types: attention quotient (ATQ), basic rhythm quotient (BRQ), and stress resistance quotient (SQ) to determine the self-management skills by stress. ATQ, BRQ, and SQ are defined by terms applied in many previous studies on the effect of neurofeedback (Byun, 2011; Shin, 2020; Bak, 2007).

ATQ is a quotient representing the brain arousal by ratio of $\theta$ waves and SMR and age or resistance to stress. ATQ has a fixed standard by age, and the ratio (r) varies among ages. Normal adults aged 15 to 45 have approximately twice stronger $\theta$ waves than SMR, but if stronger than that, the brain function is deteriorated. This study showed that the theta waves of maladjusted soldiers are more than twice stronger, and thus their brain is not awake and lacks function. Leaving this in this condition for a long time causes problems in cognitive skills, which deteriorates sociality and makes them narrow-minded (Park, 2005). Therefore, to reduce excessive $\theta$ waves in arousal, it is desirable to make long-term plans for exposure time and period (Byun, 2017). The strength of brain waves used $(\mathrm{mV})$ is a quantified quotient showing the pretest and posttest ratios (r) of ATQ already set on the Brain Analysis ver. 1.3 program (Byun, 2017). For ATQ ratio (r), we quantified $\theta$ and SMR obtained from the Brain Analysis ver.1.3 program and used them in the calculation. The formula is as follows.

$$
\text { ATQ ratio }(\mathrm{r})=\theta / \mathrm{SMR}
$$

People with high ATQ indicates that they have high resistance or immunity to diseases or stress (Bak, 2007).

BRQ is a quotient representing brain stability. It shows brain waves of $\alpha$ waves with eyes closed, and people with high brain waves with eyes closed or relaxed and aware have high brain development and stability (Park, 2005).

SQ is a quotient that represents physical and mental fa- 
tigue due to internal and external environmental factors, calculated by the interconnection between $\delta$ waves and $\beta$ waves (Byun, 2011). For brain waves of $\beta$-low and $\beta$-hi, it is decided that high beta should be under 1 .

Moreover, the low beta being more than twice higher than high beta enables the subjects to adjust their stress and display their functions in mental activities (S.Larsen, 2012; J.Robbins, 2000). High stress indicates high fatigue and low resistance to diseases, and since stress index represents the stress resistant quotient, higher values are better. On the other hand, if the brain is overloaded, there is high beta that deteriorates brain efficiency as well as concentration, thereby putting the subjects under a lot of stress (Lee, 2012).

\section{Stress-coping mechanism}

Stress coping refers to dealing with a tough situation, and with various stress-coping mechanisms, stress does not lead to negative state or continuous stress (Kostelnik et al., 2017). Moreover, stress that feels like a threat affects coping mechanisms, and excessive stress deteriorate the cognitive functions and the ability to process information, thereby disturbing problem-centered coping (Lazarus and Folkman, 1984). Therefore, if individual soldiers have the skills to effectively cope with a stressful situation with behavioral or cognitive coping efforts to handle conflicts between environment and internal needs, the current situation will no longer seem negative (Yi, 1999).

The stress coping scale revised by Kim (1987) is revised and supplemented by Sim (1995), increasing clinical utility of the stress coping scale. The scale is comprised of total 20 items: 9 items on troubleshooting, 4 items on emotional remission, 2 items on pursuit of help, 2 items on avoiding the problem, and 3 items on wishful thinking. The Cronbach's alpha of the scale was 0.92 . The items are rated on a 5-point scale, with higher stress coping scores indicating better stress coping skills. Here, the Cronbach's alpha of the stress-coping mechanism was 0.764 .

\section{Data analysis method}

We measured the variables before and after plant fragrance therapy of Green Camp soldiers who participated in the therapy once each, and data was analyzed using IBM Spss Statistics 23. The interconnection of ATQ, BRQ, and SQ was measured before and after participating in the therapy. This attempt provides all kinds of information away from the mere determination of time-series analysis, and this method is referred to as brain function analysis by Park (2005), quantifying the brain conditions as a quotient.

The paired t-test was used to analyze the mean difference in the stress-coping mechanism and self-regulation of brain waves before and after the therapy. All data was tested using the two-tailed test at the significance level of .05 and .001 , and the reliability of the scale was calculated using Cronbach's $\alpha$. Data from brain function analysis was processed by analyzing pretest-posttest brain waves $(\mathrm{mV})$ and comparatively analyzing the pretest-posttest mean differences (Byun, 2017).

\section{Results and Discussion}

Table 1 shows the results of quantifying and analyzing physiological and psychological changes by measuring brain waves before and after plant fragrance therapy for maladjusted soldiers.

As a result of analyzing ATQ before and after the therapy as shown in Table 1, both left and right brain did not show a statistically significant difference (L:t $=1.517$, $\mathrm{df}=61, p=.134 / \mathrm{R}: \mathrm{t}=0.800, \mathrm{df}=61, p=.427)$. However, the individual difference in ATQ among participants decreased from $\mathrm{SD}=2.2589$ to $\mathrm{SD}=1.3409$ after the therapy, but there was no statistically significant change.

As a result of analyzing BRQ as shown in Table 1, $\alpha$ waves with eyes closed on both left and right brain were higher in psychological and physical stability of maladjusted soldiers after the therapy, and there was statistical significance (L:t $=-3.637, \mathrm{df}=61, \mathrm{p}=.001 / \mathrm{R}: \mathrm{t}=-3.614$, $\mathrm{df}=61, p=.001)$. The fact that the mean is higher after the therapy indicates that the therapy decreased tension and was thus effective in finding stability. Here. BRQ is the quantified $\alpha$ derived from the Brain Analysis ver. 1.3 program. $\alpha$ waves, which account for about $50 \%$ of brain waves, enable subjects to sufficiently inhale volatilized plant fragrances through respiration method such as medication or 
Table 1. Comparison of EEGs Before and After Applying Plant Fragrance Therapy to Military Maladaptive Soldiers (Biological forest healing effects)

\begin{tabular}{|c|c|c|c|c|c|c|c|}
\hline Quotient & Time & $\mathrm{N}$ & $\mathrm{M}(\mathrm{mV})$ & SD & $\mathrm{t}$ & $\mathrm{df}$ & $\mathrm{p}$ \\
\hline \multirow{4}{*}{ ATQ } & PFT Before(L) & \multirow{2}{*}{62} & 4.9473 & 2.2589 & \multirow{2}{*}{1.517} & \multirow{2}{*}{61} & \multirow{2}{*}{$.134^{\mathrm{NS}}$} \\
\hline & PFT After(L) & & 4.4369 & 1.3409 & & & \\
\hline & PFT Before(R) & \multirow{2}{*}{62} & 4.6629 & 1.4368 & \multirow{2}{*}{.800} & \multirow{2}{*}{61} & \multirow{2}{*}{$.427^{\mathrm{NS}}$} \\
\hline & PFT After(R) & & 4.4642 & 1.3348 & & & \\
\hline \multirow{4}{*}{ BRQ } & PFT Before(L) & \multirow{2}{*}{62} & 4.4944 & 1.5869 & \multirow{2}{*}{-3.637} & \multirow{2}{*}{61} & \multirow{2}{*}{$.001 * *$} \\
\hline & PFT After(L) & & 5.4066 & 1.7772 & & & \\
\hline & PFT Before(R) & \multirow{2}{*}{62} & 4.3816 & 1.5182 & \multirow{2}{*}{-3.614} & \multirow{2}{*}{61} & \multirow{2}{*}{$.001^{* *}$} \\
\hline & PFT After(R) & & 5.2869 & 1.8029 & & & \\
\hline \multirow{8}{*}{ SQ } & PFT Before(L- $\beta$ low) & \multirow{2}{*}{62} & 1.6405 & .6454 & \multirow{2}{*}{-3.218} & \multirow{2}{*}{61} & \multirow{2}{*}{$.002 * *$} \\
\hline & PFT After(L- $\beta$ low $)$ & & 2.1919 & 1.2226 & & & \\
\hline & PFT Before(R- $\beta$ low) & \multirow{2}{*}{62} & 1.6473 & .6370 & \multirow{2}{*}{-4.010} & \multirow{2}{*}{61} & \multirow{2}{*}{$.000 * * *$} \\
\hline & PFT After(R-ßlow)) & & 2.3619 & 1.2796 & & & \\
\hline & PFT Before(L- $\beta h i)$ & \multirow{2}{*}{62} & 1.0456 & .6483 & \multirow{2}{*}{2.227} & \multirow{2}{*}{61} & \multirow{2}{*}{$.030 * *$} \\
\hline & PFT After(L- $\beta h i)$ & & .8440 & .3901 & & & \\
\hline & PFT Before(R- $\beta h i)$ & \multirow{2}{*}{62} & 1.136 & .6629 & \multirow{2}{*}{3.274} & \multirow{2}{*}{61} & \multirow{2}{*}{$.002 * *$} \\
\hline & PFT After(R- $\beta h i)$ & & .8097 & .3751 & & & \\
\hline
\end{tabular}

$* * * p<.001$ before and after the program with paired t-test.

$\mathrm{NS},{ }^{*}, * *$ Non-significant and significant at $p<.05$ respectively by paired t-test.

PFT: Plant Fragrance Therapy. L: Lift, R:Right, M: Mean, SD: Standard deviation.

ATQ: Attention Quotient. BRQ: Basic Rhythm Quotient. SQ: Stress Resistance Quotient

by relaxing with eyes closed, so that the inhaled fragrance molecules stimulate the olfactory nerves of olfactory bulbs and delivered after the synapse of the olfactory brain, through which they have a therapeutic effect on the autonomic nervous system, endocrine regulation of the immune system, and physical diseases and symptoms (Kim, 2011), thereby adjusting BRQ. Cupressus, which is a tree species found in the military camp, is the Pinales of gymnosperms that affects the respiratory system and circulatory system and helps regulate blood circulation and lymphokinesis (Wanda Sellar, 2001; Veronica Sibley, 2003). Various pharmacological actions volatilized from Cupressus sempervirens essential oil can be used in neurological disorders such as depression (Eddouks et al., 2002) by applying plant fragrance therapy to maladjusted soldiers, which implies that the fragrances encountered by soldiers help stabilize their negative psychological and behavioral dispositions.

As a result of analyzing SQ as shown in Table 1 to determine the resistance and immunity to internal and external stress of maladjusted soldiers, SQ was higher after the therapy on both left and right brain with statistical significance (L- $\beta$ low: $p=.002 / \mathrm{R}-\beta$ low: $p<.001 / \mathrm{L}-\beta$ hi: $p=.030 / \mathrm{R}-\beta$ hi: $p=.002)$. SQ is the resistance quotient to overcome stress, which is the state of psychological and physical tension, anxiety, and excitement. If brain waves are excited or unstable, rapid $\beta$ waves are activated, and $\beta$-hi should be below 1 (Jang, 2001). This study also revealed that $\beta$-hi was below 1 after the therapy (L- $\beta h i:=0.8440$, $\mathrm{R}-\beta h i:=0.8097)$, indicating that plant fragrance therapy increased immunity to psychological stress as well as physical tension and diseases. Moreover, $\beta$-low was at least twice higher than $\beta$-hi after the therapy, showing that SQ was high, which implies that the subjects can control their own stress (L- $\beta$ low : / L- $\beta$ hi $:=2.59$, R- $\beta$ low $: /$ R- $\beta$ hi $:=$ 2.91). In addition, the scent of flowers that took up $32.4 \%$ of plant fragrance preference of maladjusted soldiers was effective in stability and antidepression (Cauffield, 2001; Combest, 1999; Lis-Balchin and Hart, 1999), thereby proving effective in improving self-regulation and immunity to stress of maladjusted soldiers. 
Table 2. Comparison of stress response techniques before and after applying plant fragrance therapy to military adaptors (psychological forest healing effects)

\begin{tabular}{|c|c|c|c|c|c|c|c|c|}
\hline \multicolumn{2}{|c|}{ Groups } & Time & $\mathrm{N}$ & M & SD & $\mathrm{t}$ & df & $p$ \\
\hline \multirow{2}{*}{\multicolumn{2}{|c|}{ Troubleshooting }} & PFT Before & \multirow{2}{*}{62} & 2.290 & 1.179 & \multirow{2}{*}{-2.702} & \multirow{2}{*}{61} & \multirow{2}{*}{$.009 * *$} \\
\hline & & PFT After & & 2.790 & .833 & & & \\
\hline \multirow{2}{*}{\multicolumn{2}{|c|}{$\begin{array}{l}\text { Emotional } \\
\text { remission }\end{array}$}} & PFT Before & \multirow{2}{*}{62} & 2.242 & 1.351 & \multirow{2}{*}{-2.173} & \multirow{2}{*}{61} & \multirow{2}{*}{$.034 * *$} \\
\hline & & PFT After & & 2.726 & .926 & & & \\
\hline \multirow{6}{*}{$\begin{array}{l}\text { stress-coping } \\
\text { mechanism }\end{array}$} & The pursuit & PFT Before & \multirow{2}{*}{62} & 1.838 & 1.043 & \multirow{2}{*}{-3.286} & \multirow{2}{*}{61} & \multirow{2}{*}{$.002 * *$} \\
\hline & of help & PFT After & & 2.387 & 1.077 & & & \\
\hline & \multirow{2}{*}{$\begin{array}{l}\text { Avoiding a } \\
\text { problem }\end{array}$} & PFT Before & \multirow{2}{*}{62} & 2.048 & 1.123 & \multirow{2}{*}{0.79} & \multirow{2}{*}{61} & \multirow{2}{*}{$.938^{\mathrm{NS}}$} \\
\hline & & PFT After & & 2.032 & 1.071 & & & \\
\hline & \multirow{2}{*}{$\begin{array}{l}\text { Wishful } \\
\text { thinking }\end{array}$} & PFT Before & \multirow{2}{*}{62} & 2.145 & .973 & \multirow{2}{*}{-3.714} & \multirow{2}{*}{61} & \multirow{2}{*}{$<.001 * * *$} \\
\hline & & PFT After & & 2.807 & .902 & & & \\
\hline
\end{tabular}

$* * * p<.001$ before and after the program with paired t-test.

$\mathrm{NS},{ }^{*}, * *$ Non-significant and significant at $p<.05$ respectively by paired t-test.

PFT: Plant Fragrance Therapy. M: Mean, SD: Standard deviation.

Stress-coping mechanism in Table 2 refers to behavioral or cognitive coping efforts for maladjusted soldiers to handle the conflicts between their living environment and inner needs. Plant fragrances were used as a mechanism to effectively cope with stress. Directly affecting the olfactory brain with plant fragrances reduces stress with psychological and physiological changes inside the body, thereby facilitating coping mechanisms (Kim. et al., 2020).

There was a positive change in behavioral and cognitive coping efforts of individual soldiers after the therapy. Stress-coping mechanism is comprised of troubleshooting $(\mathrm{t}=-2.702, \mathrm{df}=61, p=.009)$, emotional remission $(\mathrm{t}=-2.173$, $\mathrm{df}=61, p=.034)$, pursuit of help $(\mathrm{t}=-3.286, \mathrm{df}=61$, $p=.002)$, and wishful thinking $(\mathrm{t}=-3.714, \mathrm{df}=61, p<.001)$, and there were statistically significant results. As such, plant fragrance therapy can be used to effectively cope with stress (Mass et al., 2009), and improving stress-coping mechanisms strengthens an individual's ability to deal with the frustrations in personal relations, solve problems, and accept challenges, thereby overcoming stress (Bai, 2006). On the other hand, the result of analyzing avoiding the problem $(p=.938)$ was not statistically significant. Plant fragrance therapy applied here was citrus essential oil that accounted for $27.02 \%$ of the plant fragrance preference of maladjusted soldiers, which both soothes and lifts up the mind, thereby having an excellent effect on anxiety, depression, and tension of nerves while also cheering up the mood, and this reduced the action of the sympathetic nervous system and relieves anger and disappointment (Cha, 2002). This is because the participants showed a change, trying to solve problems with positive communication and relationship building skills by encountering the right plant fragrances for them and getting to know the dispositions of themselves and others (Table 2).

\section{Conclusion}

This study verified the physiological and psychological effects of plant fragrance therapy to analyze whether the therapy has a forest healing effect on maladjusted soldiers. The results are as follows.

As a result of analyzing BRQ and SQ to determine the physiological and psychological healing effect of plant fragrance therapy, both the left and right brain showed higher BRQ and SQ after the therapy with statistical significance. This indicates that plant fragrance therapy on maladjusted soldiers helped positively stabilize their negative psychological and behavioral dispositions, and stabilization of brain waves lowered physical and mental stress and improved self-regulation and immunity. On the other hand, there was no significant difference in ATQ. There are low fluctuations of brain waves, and ATQ is off the normal range due to living environment issues of maladjusted sol- 
diers and psychological anxiety of individuals, but the ratio gradually decreased after the therapy and increased the number of participants within the normal range. Long-term research is additionally needed on the period of plant fragrance therapy, exposure time, and correlation with sleep.

Stress-coping mechanism refers to behavioral or cognitive coping efforts for maladjusted soldiers to handle the conflicts between their living environment and inner needs. Plant fragrances were used as a mechanism to effectively cope with stress. After the therapy, maladjusted soldiers were less stressed about military service and better ability to cope with it. This indicates that plant fragrance therapy is effective in stress-coping mechanisms of maladjusted soldiers.

When plant fragrances individually preferred by maladjusted soldiers are inhaled into the body, they stimulate olfactory nerves and are transmitted to the olfactory brain, releasing neurotransmitters, which helps the soldiers find mental stability through the parasympathetic nervous system and reduce stress through the endocrine system. Moreover, their immunity is improved through the lymphatic system. Therefore, applying plant fragrance therapy to maladjusted soldiers positively stabilized their negative psychological and behavioral dispositions and helped their self-regulation. Thus, plant fragrance therapy has psychological and physical forest healing effect on maladjusted soldiers.

There were difficulties in checking the detailed medical prescriptions of individual maladjusted soldiers and their exposure to plant fragrances in this study. Thus, further research must examine whether individual intervention of plant fragrance therapy has a long-term effect on individuals.

ATQ did not return clearly to the normal range, which was contrary to our expectations. This may be due to insufficient time and period of exposure to volatilized plant fragrances, the characteristics of the subjects, or the difference in $\theta$ waves and SMR acceptance of individual soldiers in brain wave measurement.

This study revealed that individual application of plant fragrance therapy was effective in motivating participants' interest and individual change. By diagnosing the mental and physical state of individuals and applying individual plant fragrances, participants can be interested in managing their own stress and change their negative behavior, thereby resulting in forest healing effect as an alternative therapy.

This study has limitations in that it is a comparative study within the group without the control group that it took place in a military camp in Gangwon-do. In addition, it was difficult to sufficiently use plant fragrances in various forests due to the limited space and activities, and the subjects were only male soldiers at Green Camp.

The forest healing effect on some maladaptive subjects can be expanded as maladjusted soldiers are actually negative and maladaptive to the military society and have difficulty in self-regulation and management. Accordingly, additional research must be conducted on positive physiological change of plant fragrances for individual subjects as well as activity space and period to maximize the efficiency of forest healing. Furthermore, it is necessary to explore improvement of specific physiological functions using each factor of forest healing.

\section{References}

Ait Igri, M., M. Holeman, A. llidrissi and M. Berrada. 1990. Contributions a I'etude des huiles essentielles des rameaux et du bois de Tetraclinis articuata (Vahl) Masters. Plantes Medicinal et Phytotherapie 24(1):36-43

Bai. J.S. 2006. The effect of stress self esteem and social support on depression-with the youth of the rural and finishing areas phD Dissertation. Chonnam Univ, Kwangju.

Barrero, A.F., J.F.Q. del Moral, R. Lucas, M. Paya, M. Akssira, S. Akaad, and F. Mellouki. 2003. Diterpenoids from Tetraclinis articulata that Inhibit Various Human Leukocyte Functions. J. Nat. Prod. 66(6):844-850. https://doi.org/10.1021/np0204949

Bak, K.J., S.G. Yi and P.W. Park. 2007. A Reserch on the Effect Neurofeedback Traing before \& After About Emotional and Attention Deficit Characteristics by Timeseies Linear Analysis : for Primary Student. J. Inf. Technol. Appl. Manag. 14(4):43-59.

Buhagiar, J.A., M.T. Camilleri Podesta, G. Flamini, P.L. Cioni and I. Morelli. 2000. Contribution to the chemical investigation of the essential oils extracted from leafy and woody branches. Cones and Seeds of Tetraclinis articulata (Vahl) Masters. J Essent Oil Res 12:29-32. https://doi.org/10.1080/10412905.2000.9712034 
Byun, Y.E. 2017. Effect of Prefrontal lobe Neurofeedback Training for reducing Adolescent Theta wave. J. Korea Acad. Ind. Coop. Soc. 18(12):459-465. https://doi.org/1 0.5762/KAIS.2017.18.12.459

Byun. Y.E. and P.W. Park. 2011. The effect of Neurofeedback Training on Sex differences groups in Adolescence. J. Korea Acad. Ind. Coop. Soc. 12(3):1171-1177. https://doi. org/10.5762/KAIS.2011.12.3.1171

Cha, S.H. 2002. Effects of Scent Inhalation Method Using Bergamot Essential Oil on Stress Symptoms in Middle-aged Women. Master's thesis, chungang University. Seoul. Korea.

Jang, K.S. 2007. The Influencing factors of adjusting to military life for new generation soldiers based on self esteem and stress. Master's thesis, Soongsil University, Seoul, Korea

Cauffield, J.S. 2001. Supplements Used to Treat Sleep Disorders. U. S. Pharmacist, 26(6).

Combest, W.L. 1999. Lavender: This herb's properties include sedative, antimicrobial and anticonvulsant effects. U. S. Pharmacist, 24(8):24-37

Development of Therapeutic Program on Forests. 2011. Korea Forest Service. PRN 11-1400000-000503-0

Eddouks M., M. Maghrani, A. Lemhardi, M.L. Ouahidi, and H. Jouad. 2002. Ethnopharmacological survey of medicinal plants used for the treatment of diabetes mellitus, hypertension and cardiac and diseases in the south-east region of Morocco(Tafilalet). J Ethnopharmacol. 82(2-3): 97-103. https://doi.org/10.1016/s0378-8741(02)00164-2

Fabricant, D.S, and N.R. Farnsworth. 2001. The Value of Plants Used in Traditional Medicine for Drug Discovery. Environ. Health Perspect. Vol.109(Suppl. I):69-76. https://doi.org/10.1289/ehp.01109s169

Groombridge Brian, 1992, Global biodiversity : status of the earth's living resources : a report. Chapman \& Hall, London, New York.

Ha, A.R. 2006. Effects of Aromatherapy on Health Promotion by Using Electroencephalogram Spectra and the User's Behavior. Doctoral dissertation, Kosin University, Busan, Korea.

Hamilton, A., K. Dürbeck and A. Lawrence. 2006, TOWARDS A SUSTAINABLE HERBAL HARVEST A work in hand. Plant Talk, Issue 43(pp. 32-35).

J.K. Jung, and Han, Y.H. 2007. Two-factor Self-Esteem
Scale: Individualistic and Collectivistic Factors. Korean J. Soc. Pers. Psychol. 21(4):117-131. https://doi.org/10.2 1193/kjspp.2007.21.4.007

Kim. Y.J and Chang. N.K. 2001. Changes of the Prefrontal EEG (Electroencephalogram) Activities according to the Repetition of Audio-Visual Learning. J. Korean Assoc. Sci. Educ. 21(3):516-528.

Joe, S.Y. 2003. Relations of health promoting behaviors, mental health, and millitary life adjustments of soldiers. J. Korea Acad. Psychiatr. Ment. Health Nurs. 12(2): 164-171.

Jun. Y.S., J.S. Kim, and C.M. Park. 2007. The Influence of Beauty Education Program the Disabled Women. J. Korean Soc. Cosmetol. 13(3):989-1000.

Jung, S.G. 2013. Military Service Stress and Psychological Maladjustment : The Mediating Role of Child modes. Master's thesis, Kyungnam University, Chang Won, Korea. http://www.riss.kr/link?id=T13243994

Jung, H.J. 2007. The Stress Coping Style and Military Life Adjustment of Soldiers. Master's thesis, Kyungbuk University, Daegu, Korea.

Kim. J.H. 1987. The action on perceived stress, cognitive and coping depression. Seoul National University.

Kim. J.H., C.S. Sin, J.h Kim, D.H. Kim, Y.S. Kim, H.C. Lee. 2020. The Stress-Reducing Effects of Forest Healing Activities in Maladjusted Military Force Members. J. People Plants Environ. 23(3):377-385. https://doi.org/1 0.11628/ksppe.2020.23.3.377

Kim. Y.J. 2000. Development of brain Circulation learning Model based on EEG Analysis of learning Activities. Master's thesis, Seoul National University, Seoul, Korea.

Kim, Y.N. 2011. The study on effect of anti-stress managed by aroma essence oil therapy through oldfactory. Master's thesis, Kyonggi University, Seoul, Korea.

Kogan, N.H., and P.A. Betrus. 1984. Self-management: A nursing mode of therapeutic influence. ANS Adv. Nurs. Sci. 6(4):55-73.

Kostelnik, M.J., A. Soderman, A. Whiren, M.L. Rupiper, and K. Gregory. 2015. Guiding children's social development and learning(8th ed.). Boston, MA: Cengage Learning US.

Kris-Etherton, P.M., K.D. Hecker, A. Bonanome, S.M. Coval, A.E. Binkoski, K.F. Hilpert, A.E. Griel, and T.D. 
Etherton. 2002. Bioactive compounds in foods: their role in the prevention of cardiovascular disease and cancer. Am. J. Med. Vol.113 (Suppl. I): 9(71-88). https://doi.org/10.1016/S0002-9343(01)00995-0

Larsen, S. 2012. The Neurofeedback Solution : How to Treat Autism, ADHD, Anxiety, Brain lnjury, Stroke, PTSD, and More. Healing Arts Press: Original ed. edition.

Lazarus, R. and S. Folkman. 1984. Stree, appraisal, and coping. Springer Publishing Co.

Lee. B.K. and H.H. Lee. 2012. A Study on the Effects of Human Physiology after Forest Phytoncide Therapy. J. Naturopathy. 1(1):14-20.

Lee, B.K. 2012. A Study on the Effects of Naturopathy after Forest Phytoncide Therapy. Doctoral dissertation, Dongbang Culture University, Seoul, Korea.

Lee, K.J. 2003. Psychological characteristics of high-risk and low-risk groups of juvenile suicide. Master's thesis, The Catholic University, Kyonggi, Korea.

Lee, I.H. 1999. Effects of Stress and Coping on Body Perception. Korean J. Health Psychol. 4(1):80-93

Lee, Y.J. 2003. (The) Effects of Lavender Aromatherapy on Sleep and Depression of Elderly. Doctoral dissertation, Chungnam University, Daejeon, Korea.

Lis-Balchin, M. and S. Hart, 1999. Studies on the mode of action of the essential oil of LavenderLavandula angustifolia P. Miller). Phytother. Res. 13(6):540-542. https://doi.org/10.1002/(sici)1099-1573(199909)13:6<5 40::aid-ptr523>3.0.co;2-i

Lubar. J.F. 1995. Neurofeedback for the management Attention-deficit/hyperactivity disorders. In M. SSchwartz (Ed.), Biofeedback:A practitioner's guid New York: Guilford Press.(pp.493-522)

Mass, G., S. Ulirch, C. Hornberg and T. Classen. 2009. Frische Waldluft fur die Mitarbeiter! Waldtage als Stress reduzierende Handlungsstrategie in der betrieblichen Gesundheitsforderung.In:Eidg. Forschungsanstalt fur Wald, Schnee und Landschaft(WSL)(Hrsg.), Konferenzdokumentation:Gesundheit und Erholung in Wald und Landschaft. Birmensdorf, Swiss. (pp. 53).

Nam, E.S. and D.C. Uhm. 2008. Effects of Phytoncides Inhalation on Serum Cortisol Level and Life Stress of College Students. J. Korean Acad. Soc. Adult Nurs. 20(5):697-706.

Miller, L.S., B. Colella, D. Mikulis, J. Maller, and R.E.A.
Green. 2013. Environmental Enrichment May Protect Against Hippocampal Atrophy in the Chronic Stages of Traumatic Brain lnjury. Front. Hum. Neurosci. 7:506. https://doi.org/10.3389/fnhum.2013.00506

Order of Military Management, 2019, Order of Defense (Act No.2273). Korea. Retrieved from http://www.law.go.kr

Park, H.S., C.S. Shin, P.S. Yeon, and J.Y. Kim. 2014. A Comparative Study on the Stress Recovery Effect of Forest Therapy. J. Korean Inst. For. Recreat. 18(1):13-24. https://doi.org/10.34272/forest.2014.18.1.002

Park, P.W. 2005. Introduction to Neurofeedback: Based on the use of NeuroHarmony. Seoul, Korea : Incorporated foundation Korea Institute of Psychiatry.

Robbins, J. 2000. The Evolution of the New Brain Wave Biofeedback. A Symphony in the Brain, New York : Grove Press.

Rosenberg, M. 1965. Society and the adolescent self-image. Princeton NJ : Princeton University Press.

Schippmann, U., D. Leaman and A.B. Cunningham. 2006. Cultivation and wild collection of medicinal and aromatic plants under sustainability aspects. Medicinal and Aromatic Plants: Agricultural, Commercial, Ecological, Legal, Pharmacological and Social Aspects. (17). https://librar y.wur.nl/ojs/index.php/frontis/issue/view/232

Shin, J.E., Y.G. Kim, and H.W. Weon. 2020. Effect of Prefrontal Neurofeedback Training on the Attention and of Adolescent. J. Korea Acad. Ind. Coop. Soc. 21(3): 447-452. https://doi.org/10.5762/KAIS.2020.21.3.447

Sim, S.J. 1995. (The) effect on stress level by assertiveness and the way of stress coping. Master's thesis, The Catholic University, Kyonggi, Korea.

Song, S.Y. 2015, (The) Effects of Phytoncide Aromatherapy on Work Stress and Mental Health of Women at Insurance Call Center. Master's thesis, Kyonggi University, Seoul, Korea.

Sibley, V. 2005. Aromatherapy Solutions. Hamlyn; 0 edition.

Sellar, W. 2001. The Directory of Essential Oils. The C.W. Danial. Random House UK; New Ed edition

You, J.H and W.S. Shin. 2017. The Infiuence of Green Camp on Soldiers' Mood States and Military Stress. J. Korean Inst. For. Recreat. 21(4):1-8. https://doi.org/10.3 4272/forest.2017.21.4.001

Yi, I.H. 1999. Effects of stress and coping on body perception. Korean J. Health Psychol. 4(1):80-93. 